\title{
O papel do estresse oxidativo na Doença de Crohn: Uma revisão narrativa
}

\author{
The role of oxidative stress in Crohn's disease: A narrative review \\ El papel del estrés oxidativo en la enfermedad de Crohn: Una revisión narrativa
}

Recebido: 30/03/2021 | Revisado: 09/04/2021 | Aceito: 12/04/2021 | Publicado: 21/04/2021

\author{
Fernanda da Silva Basílio \\ ORCID: https://orcid.org/0000-0003-1975-7655 \\ Universidade de Caxias do Sul, Brasil \\ E-mail: fsabasilio@ucs.br \\ Júlia Maiara dos Santos \\ ORCID: https://orcid.org/0000-0002-7776-7555 \\ Universidade de Caxias do Sul, Brasil \\ E-mail: jmsantos7@ucs.br \\ Cátia Santos Branco \\ ORCID: https://orcid.org/0000-0003-3709-3004 \\ Universidade de Caxias do Sul, Brasil \\ E-mail: csbranc1@ucs.br
}

\begin{abstract}
Resumo
A doença de Crohn (DC) faz parte de um conjunto de desordens inflamatórias do trato gastrointestinal de sintomatologia variada e caráter crônico. Evidências têm mostrado uma associação entre estresse oxidativo e DC. Nesse sentido, diferentes biomarcadores estão sendo estudados, porém ainda não há consenso. O objetivo deste estudo foi compilar os mais recentes estudos sobre o equilíbrio oxidante/antioxidante na DC em pacientes ativos e em remissão. Dentre os biomarcadores, os relacionados à peroxidação lipídica foram os mais estudados na DC. Observou-se que, mesmo com a doença sob controle, pacientes remissivos continuam a apresentar níveis de estresse oxidativo (danos oxidativos aos lipídeos e proteínas) altos e defesas antioxidantes (atividade de GPx e catalase) alteradas. Além disso, os níveis plasmáticos de selênio, um mineral com importante ação antioxidante, foram encontrados diminuídos nos pacientes, independentemente do estágio da doença. Esses achados demonstram a necessidade de monitorar o paciente em relação aos biomarcadores de estresse oxidativo e de defesa antioxidante, especialmente em períodos de remissão, em que o paciente não apresenta sintomas, porém mantém as alterações oxidativo-inflamatórias latentes. Os dados aqui discutidos poderão colaborar para a concepção e desenvolvimento de novas abordagens para pacientes com DC.
\end{abstract}

Palavras-chave: Doenças inflamatórias intestinais; Antioxidantes; Vitaminas; Minerais.

\begin{abstract}
Crohn's disease (DC) is part of a set of inflammatory disorders of the gastrointestinal tract of varied symptoms and chronic character. Evidence has shown an association between oxidative stress and DC. Therefore, different biomarkers are being studied, but there is still no consensus. This study aimed to compile the most recent studies on the oxidant/antioxidant balance in DC in active and in remission patients. Among the biomarkers, those related to lipid peroxidation were the most studied in DC. It was observed that, even with the disease under control, remissive patients continue to present high levels of oxidative stress (oxidative damage to lipids and proteins) and alterations in their antioxidant defenses (GPx and catalase activities). Moreover, plasmatic levels of selenium, a mineral with important antioxidant action, were found to be decreased in patients, regardless of the disease's stage. These findings demonstrate the need to monitor the oxidative stress and antioxidant defense biomarkers in DC patients, especially in periods of remission, when the patient does not have symptoms but maintains latent oxidative-inflammatory changes. The data discussed here may contribute to the design and development of new approaches for patients with DC.
\end{abstract}

Keywords: Inflammatory bowel diseases; Antioxidants; Vitamins; Minerals.

\section{Resumen}

La enfermedad de Crohn (DC) forma parte de un conjunto de trastornos inflamatorios del tracto gastrointestinal de variada sintomatología y carácter crónico. La evidencia ha demostrado una asociación entre el estrés oxidativo y la DC. Por tanto, se están estudiando diferentes biomarcadores, pero aún no hay consenso. Este estudio tuvo como objetivo recopilar los estudios más recientes sobre el equilibrio oxidante/antioxidante en DC en pacientes activos y en remisión. Entre los biomarcadores, los relacionados con la peroxidación lipídica fueron los más estudiados en DC. Se observó que, incluso con la enfermedad bajo control, los pacientes remisivos continúan presentando altos niveles de estrés oxidativo (daño oxidativo a lípidos y proteínas) y alteraciones en sus defensas antioxidantes (actividad de GPx y catalasa). Además, se encontró que los niveles plasmáticos de selenio, un mineral con una importante acción 
antioxidante, disminuían en los pacientes, independientemente del estadio de la enfermedad. Estos hallazgos demuestran la necesidad de monitorear el estrés oxidativo y los biomarcadores de defensa antioxidante en pacientes con DC, especialmente en períodos de remisión, cuando el paciente no presenta síntomas, pero mantiene cambios oxidativo-inflamatorios latentes. Los datos discutidos aquí pueden contribuir al diseño y desarrollo de nuevos enfoques para pacientes con DC.

Palabras clave: Enfermedades inflamatorias del intestino; Antioxidantes; Vitaminas; Minerales.

\section{Introdução}

As Doenças Inflamatórias Intestinais (DII) são distúrbios autoimunes que apresentam resposta imunológica deficitária ou exacerbada, sendo caracterizadas por afecções sistêmicas inflamatórias localizadas no trato gastrointestinal (TGI) (Ramos \& Papadakis, 2019). No mundo, mais de 5 milhões de pessoas sofrem com DII (ABCD, 2020) e a Doença de Crohn (DC) é uma das mais frequentes (Sairenji et al, 2017).

De acordo com a Associação Brasileira de Colite Ulcerativa e Doença de Crohn (ABCD, 2020), existem poucos dados estatísticos sobre a epidemiologia da DC no Brasil. No entanto, as evidências apontam para um crescimento expressivo na incidência da doença no país, especialmente na região sudeste (Errante \& Romano Jr., 2016).

A DC é uma doença multifatorial, ou seja, fatores hereditários e influências ambientais, dentre as quais a própria microbiota intestinal e a presença de um possível agente entérico infeccioso podem contribuir sinergicamente para o surgimento e a progressão da doença (Aleksandrova, Romero-Mosquera \& Hernandez, 2017). Tais fatores são capazes de desregular o equilíbrio entre os antígenos luminais e a resposta imune da mucosa intestinal, ativando uma cascata inflamatória (Reddavide et al, 2018).

Por se tratar de um distúrbio que apresenta uma multiplicidade de sinais e sintomas, o diagnóstico correto e seu tratamento específico ainda são empecilhos que precisam ser contornados. A causa exata da doença permanece, até o momento, desconhecida. No entanto, evidências indicam que a dieta e o estresse podem contribuir para seu surgimento (Cavalcante et al, 2020). Ademais, tem sido mostrado que a deficiência de vitaminas e minerais antioxidantes tem sido associada à DC, embora os mecanismos exatos dessa relação não estejam até o momento esclarecidos (Tumani, Pavez \& Parada, 2020).

Para conter a geração exagerada das espécies reativas de oxigênio (EROs) é ativado o sistema de defesa antioxidante, que pode ser enzimático ou não-enzimático. Evidências anteriores indicam que a ingestão de antioxidantes dietéticos poderia auxiliar na prevenção e/ou tratamento de doenças inflamatórias intestinais, tais como a DC (Volp et al, 2010; Gentschew et al 2012; Alzoghaibi, 2013).

Nesse sentido, o objetivo desta revisão foi compilar os mais recentes dados publicados sobre estresse oxidativo e antioxidantes em pacientes remissivos ou ativos portadores de DC, buscando compreender sua relação com a doença.

\section{Abordagem Metodológica}

Os estudos foram selecionados a partir da pesquisa no banco de dados Medline/PubMed. Foram incluídos artigos publicados no período de 2008 a 2020 nos idiomas inglês, espanhol e português, envolvendo seres humanos.

Foi utilizada uma combinação de um ou mais dos seguintes indexadores: doença de Crohn, inflamação intestinal, desequilíbrio redox, antioxidantes, vitaminas e minerais.

Os termos citados deveriam aparecer no título, nas palavras-chave ou no resumo do trabalho. Conforme demonstrado na Figura 1, após a pesquisa preliminar (passo 1), 34 estudos tiveram o resumo analisado (passo 2) e apenas aqueles que contemplavam o escopo principal da discussão ora proposta foram selecionados (passo 3).

Assim, foram incluídos 9 estudos dos últimos 12 anos, os quais foram analisados e utilizados para construir a lógica 
da presente revisão narrativa.

Figura 1 - Etapas de busca e seleção dos estudos para essa revisão.

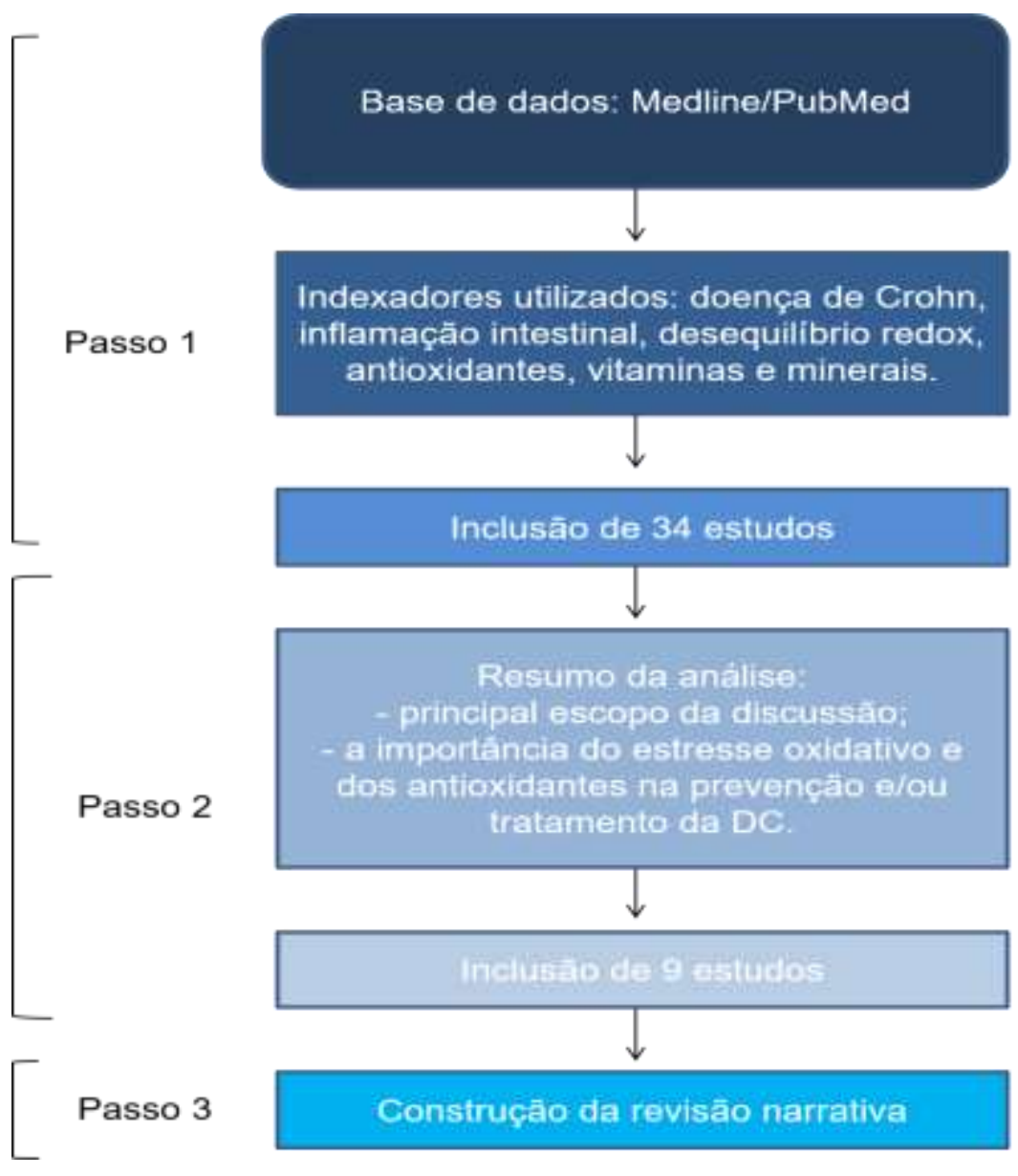

Fonte: Autores (2021).

\section{Desenvolvimento}

\section{Doenças inflamatórias intestinais (DII)}

As DII constituem um grupo de distúrbios inflamatórios que envolvem o TGI e o sistema imunológico, sob influência de fatores ambientais e genéticos, sendo esses últimos desencadeados por mutações. Existem dois subtipos de DII, a Colite Ulcerativa e a Doença de Crohn (DC), as quais ocorrem no intestino grosso e delgado, respectivamente (Neurath, 2019).

As DII acometem mais frequentemente adolescentes e jovens adultos, porém podem surgir em qualquer faixa etária (Errante \& Romano Jr., 2016). Além disso, evidências apontam uma diferença na incidência das DII entre os sexos, existindo maior prevalência da DC em mulheres, enquanto os homens são mais acometidos pela Colite Ulcerativa (Goodman, Erkkila \& Pizarro, 2020).

A incidência das DII tem aumentado consideravelmente, possivelmente em função da urbanização, baixa ingestão de fibras e aumento do consumo de carnes na dieta (Alatab et al, 2020). Ademais, dados coletados em escala global evidenciam o aumento da prevalência das DII em países emergentes nos continentes Africano, Asiático e Sul Americano, incluindo o Brasil (Ng et al, 2017). 
O papel da dieta do indivíduo também tem ganhado atenção, visto que $85 \%$ dos pacientes acometidos sofrem de desnutrição (Weisshof \& Chermesh, 2015). Percebe-se que a diversidade da dieta moderna pode colaborar para o aumento de episódios inflamatórios no TGI, especialmente quando estimulada por alimentos ultraprocessados, comumente ricos em sódio, açúcares e aditivos não naturais, podendo afetar a função e eficiência das barreiras mucosas e imunológicas (Owczarek et al, 2016).

O consumo crescente desse tipo de alimento tem sido associado com o surgimento de intolerâncias alimentares, as quais são especialmente frequentes em indivíduos com DII (Brown, Rampertab \& Mullin, 2011). Tais intolerâncias podem causar obstruções parciais e/ou alterações no trânsito gástrico-intestinal tendo como resultado má absorção, ocorrência de diarreia, alterações na produção de secreções, entre outros desfechos (Spiller \& Thompson, 2012).

$\mathrm{O}$ aumento da resposta inflamatória, a qual gera um quadro persistente e de curso crônico, resulta em danos celulares à lipídeos, proteínas e ao DNA, com diferentes graus de comprometimento tecidual (Guan \& Lan, 2018). Em quadros de remissão esses eventos são minimizados, porém em função da resposta imune exacerbada na fase aguda, os danos teciduais permanecem (Pariente et al, 2011).

\section{Doença de Crohn}

A DC tem prevalência estimada de 130 para cada 100 mil indivíduos. Podem existir dois picos em faixas etárias distintas, o primeiro entre 20 e 40 anos e o segundo entre 50 e 60 anos (Errante \& Romano Jr., 2016; Ferré, Boscá-Watts \& Pérez, 2018). Apresenta sintomas como diarreia, febre, anemia, perda de peso, intolerâncias alimentares e déficit de crescimento (no caso de acometimento de crianças e adolescentes), sendo que seus sintomas extra-intestinais são característicos dos casos de maior gravidade (Alzoghaibi, 2013).

Tem sido mostrado que, conforme aumenta a sua duração, o risco de malignidade aumenta significativamente, não apenas em função da manutenção do status inflamatório crônico, como também pela depleção na absorção de nutrientes, sendo a desnutrição, por exemplo, um de seus desfechos mais comuns (Weisshof \& Chermesh, 2015).

É importante que sejam solicitados diversos exames, como os descritos na Tabela 1, para que o diagnóstico seja o mais assertivo possível, já que outras desordens podem manifestar sintomas similares aos da DC.

Tabela 1 - Exames que podem ser solicitados para o diagnóstico da DC.

\begin{tabular}{ccc}
\hline Tipos de exame & Descrição & Referências \\
\hline Imagem & $\begin{array}{c}\text { Endoscopia, colonoscopia, raio x contrastado, } \\
\text { tomografia computadorizada, enteroressonância, } \\
\text { ultrassom abdominal }\end{array}$ & Chan, Mourad \& Leong, 2018 \\
Laboratorial & $\begin{array}{c}\text { Hemograma (para avaliação de leucocitose e } \\
\text { anemia), determinação de albumina sérica, teste } \\
\text { de velocidade de hemossedimentação, proteína C } \\
\text { reativa, exames de fezes, anticorpos } \\
\text { Biópsia proveniente de colonoscopia }\end{array}$ & $\begin{array}{c}\text { Flynn \& Eisenstein, 2019 } \\
\text { Seyedian, Nokhostin, \& Malamir, } 2019\end{array}$ \\
Histológico & Fonte: Autores (2021). & Roda et al, 2020 \\
As características da DC incluem uma distribuição descontínua das lesões, alternando entre segmentos não inflamados e & \\
inflamados. Além disso, há a presença de abcessos, fibrose, fístulas, espessamento da submucosa, estenoses localizadas, \\
segmentos estreitados e/ou obstrução parcial ou total do lúmen intestinal (Hendy \& Hart, 2013).
\end{tabular}


A DC pode ser consequência de uma interação complexa entre suscetibilidade genética, pré-disposição à disbiose e fatores ambientais, conforme mostrado na Figura 2. Tomados em conjunto, esses eventos contribuem para a ativação de respostas imunes inatas e adaptativas desreguladas (Petagna et al, 2020).

Em relação aos fatores genéticos, tem sido mostrado que a homozigosidase do gene NOD2 aumenta de 20 a 40 vezes o risco de desenvolver DC (Ananthakrishnan, 2015; Veauthier \& Hornecker, 2018). Receptores NOD2, presentes na superfície dos monócitos, desempenham um importante papel na imunidade inata contra patógenos e na regulação da imunidade adaptativa e estão mutados na DC (Errante \& Romano Jr., 2016).

Além da genética, alterações na microbiota que favorecem a ocorrência de disbiose na DC são especialmente devidas à abundância relativa de bactérias do filo Bacteroidetes e da família Enterobacteriaceae (ex.: Eschericia coli), e da depleção de bactérias do filo Firmicutes e do gênero Faecalibacterium (ex.: Faecalibacterium prausnitzii) (Wright et al, 2015).

Os fatores ambientais associados ao aumento do risco incluem o ambiente urbano, junk foods e o tabagismo, além do uso de medicações como anticoncepcionais orais, antibióticos e anti-inflamatórios não esteroidais (AINES) de uso prolongado (Ananthakrishnan, 2015).

Fatores ambientais associados à diminuição do risco da DC incluem o aleitamento materno, exposição a animais (de estimação e de fazenda), possuir irmãos, compartilhamento de quarto, além da alta ingestão de fibras, consumo de frutas e atividade física (Torres et al, 2017). 
Figura 2 - Fatores associados ao desenvolvimento da Doença de Crohn.

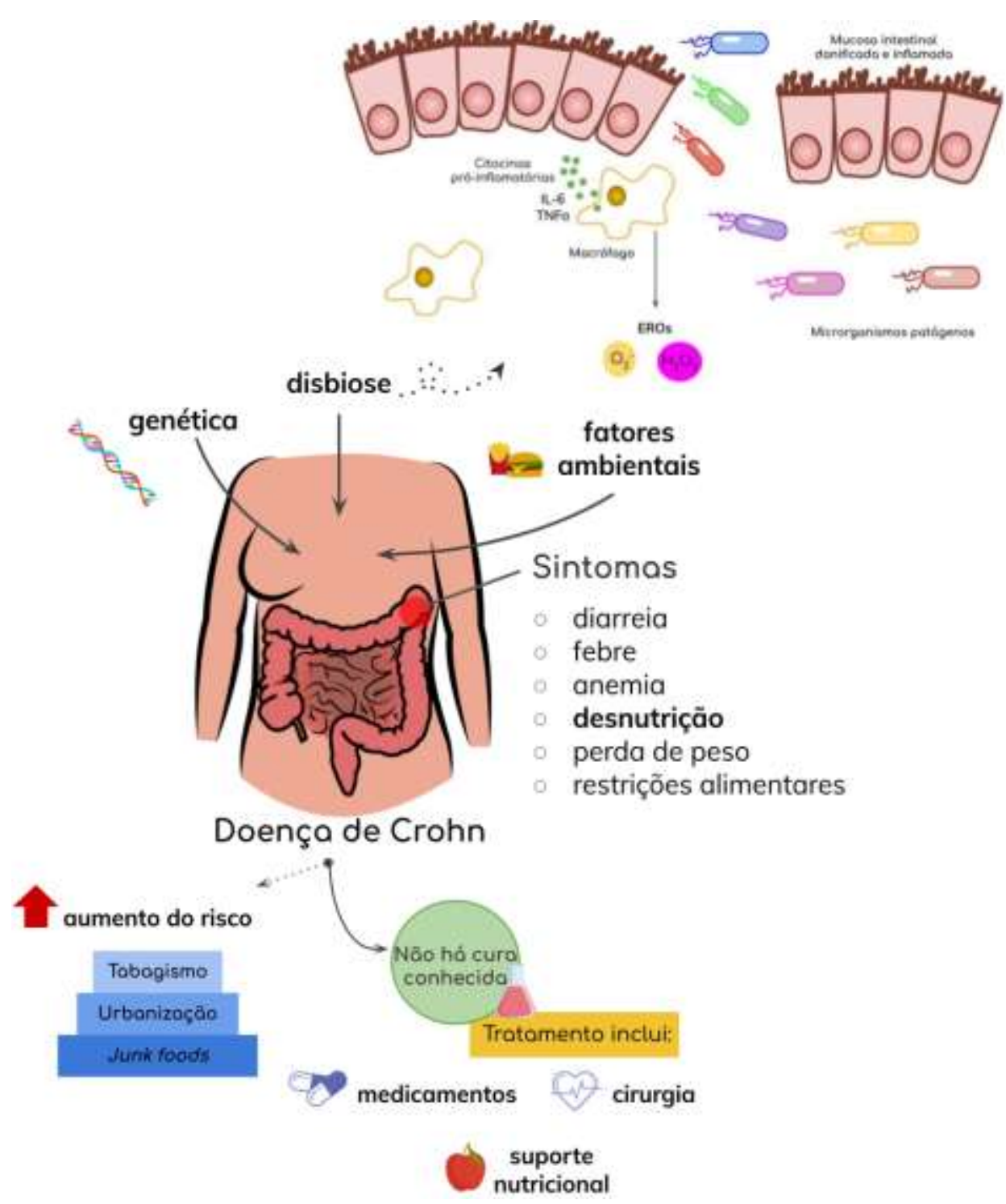

Fonte: Autores (2021).

\section{Tratamento da DC}

A intervenção medicamentosa tem como objetivo induzir e manter a patologia em remissão, visando também melhorar o estado nutricional do paciente. É de grande importância o diagnóstico precoce a fim de iniciar o tratamento específico das manifestações primárias, dessa forma minimizando o agravamento dos sintomas intestinais e extra-intestinais.

O tratamento farmacológico da DC é semelhante ao de outras DII e inclui a administração de corticosteroides, antiinflamatórios (aminossalicilatos), imunossupressores (azatioprina, ciclosporina, mercaptopurina) e antibióticos (ciprofloxacina e metronidazol). Outrossim, pode-se também fazer uso de imunobiológicos, os quais atuam como anticorpos antagonistas do Fator de Necrose Tumoral (anti-TNF) nos casos de maior gravidade (Veauthier \& Hornecker, 2018).

Além da terapia medicamentosa, cerca de 50 a $70 \%$ dos pacientes DC podem necessitar de intervenção cirúrgica, seja para a reparação de estenoses ou para a remoção de partes do intestino. Dados recentes indicam, no entanto, um alto índice de refração pós-cirúrgico em até 90 \% dos casos, ocorrendo ainda no primeiro ano (Mariosa, Chebli \& Chebli, 2011). 
Em função das limitações exibidas, tanto pelos medicamentos quanto pelos procedimentos cirúrgicos, novas propostas terapêuticas precisam ser pensadas. Nesse contexto, é de suma importância o suporte nutricional permanente, uma vez que o comprometimento nutricional é a característica mais marcante dessa condição (Santarpia et al, 2019). Isso ocorre em função da redução na ingestão alimentar devido aos sintomas gastrointestinais presentes, mas também pode ser consequência da resposta inflamatória exacerbada e suas complicações (Owczarek et al., 2016).

Diferentes abordagens, incluindo o uso de suplementos, e/ou nutrição enteral e parenteral tem se mostrado efetivas nesse sentido, porém a adequação do tratamento de suporte depende do estado atual da doença, ou seja, se ativa ou em remissão (Lichtenstein et al, 2018). Embora efetivos, a dieta e o uso de nutrientes específicos servem como uma medida adjuvante individualizada, que visa manter o estado nutricional saudável do paciente. Adicionalmente, podem auxiliar na redução dos sintomas, melhorando sua qualidade de vida (Goens \& Micic, 2020).

A comunidade científica tem se empenhado para desenvolver novas estratégias para os pacientes em ambas as fases da doença. Nesse sentido, opções nutracêuticas como o uso de ácidos graxos poli-insaturados (PUFA) da série do ômega 3, por exemplo, têm se mostrado promissores. Alimentos e/ou suplementos à base de prebióticos e/ou probióticos também têm sido propostos (Kumar et al, 2016). Além disso, hipotetiza-se sobre o papel benéfico dos antioxidantes na DC, tanto através da redução do risco da sua incidência (atuando como agentes preventivos), quanto para o seu tratamento (agindo como agentes terapêuticos).

\section{Estresse Oxidativo e Antioxidantes na DC}

Espécies reativas de oxigênio (EROs) são comumente geradas como subprodutos em diversos sistemas biológicos, tais como o metabolismo do oxigênio, sendo a mitocôndria seu principal sítio de formação intracelular (Branco et al, 2015). Essas moléculas são essenciais para o funcionamento de inúmeros processos biológicos, e, portanto, são mantidas em níveis basais. Diferenciação celular, apoptose, resposta imune e fosforilação proteica são apenas alguns exemplos de processos dependentes das EROs (Pizzino et al, 2017).

Essas substâncias podem apresentar um elétron desemparelhado em sua última camada de valência (radicais livres) tais como o radical ânion superóxido $\left(\mathrm{O}_{2}{ }^{*}\right)$ e o radical hidroxila $\left(\mathrm{OH}^{*}\right)$, mas podem não ser radicalares, e ainda assim apresentar alto potencial oxidativo, como o peróxido de hidrogênio $\left(\mathrm{H}_{2} \mathrm{O}_{2}\right)$ (Sies, 2018). Quando ocorre um aumento na produção desses metabólitos, efeitos danosos às estruturas celulares importantes (proteínas, lipídeos, ácidos nucleicos) começam a surgir (Branco et al, 2015).

Além da produção endógena, o organismo pode ser afetado pelas EROs exógenas, através da exposição ambiental (Warraich, Hussain \& Kayani, 2020). Conforme demonstrado na Figura 3, o estresse oxidativo ocorre quando há um desequilíbrio na geração de EROs (pró-oxidante) frente à capacidade de defesa antioxidante da célula. A defesa, endógena ou exógena, tem o papel de inibir e/ou minimizar os agravos causados pela ação deletéria dessas espécies (Smits et al, 2019).

Dentre as defesas endógenas pode-se citar a superóxido dismutase (SOD), catalase (CAT), glutationa peroxidase (GPx), classificadas como componentes enzimáticos, ácido lipoico, ácido úrico e bilirrubina, classificados como não enzimáticos. Já entre os antioxidantes exógenos, pode-se destacar os carotenoides, as vitaminas E, A e C, entre outros (Neha et al, 2019).

Essas substâncias podem ser administradas por via oral como um suplemento único ou em combinação. No entanto, o monitoramento de seus níveis séricos é importante na DC, especialmente em função do prejuízo na absorção durante a fase aguda da doença. 
Figura 3 - Mecanismo antioxidante de controle de EROs. Em situação de desequilíbrio, as EROs se acumulam e podem causar danos à diversas estruturas celulares, como lipídeos de membrana, proteínas e material genético.

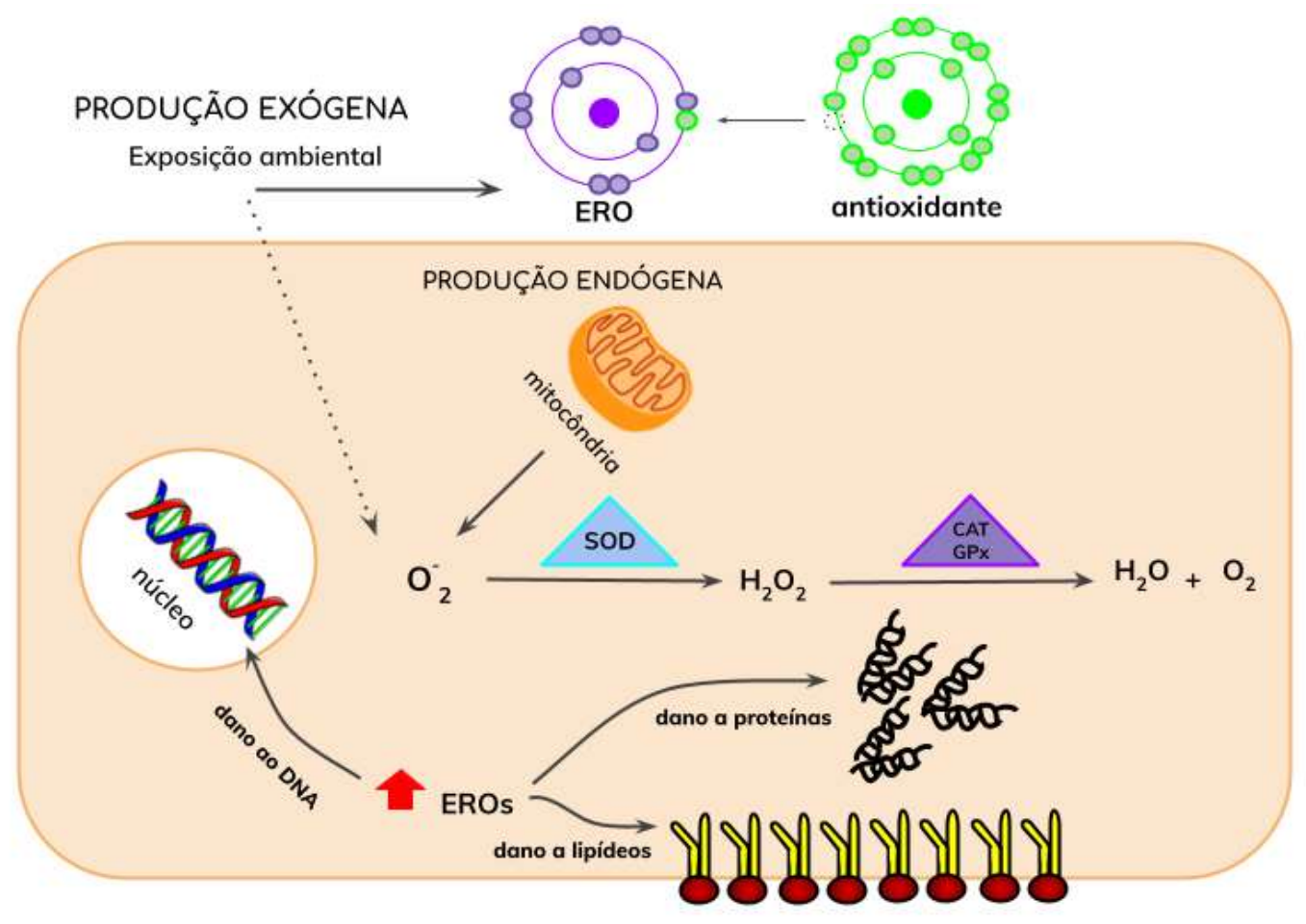

Fonte: Autores (2021).

A inflamação, principal característica patológica das DII, é um processo fortemente ligado à geração de espécies reativas de nitrogênio (ERNs) e EROs. Na DC observa-se a entrada expressiva de células inflamatórias na mucosa afetada. Os neutrófilos e macrófagos que chegam estimulam a produção de EROs, as quais são potencialmente prejudiciais, já que podem levar ao estresse oxidativo (Alemany-Cosmi et al, 2021), como previamente discutido.

Biomarcadores de estresse oxidativo vem sendo empregados em diversas doenças crônicas, dentre elas a DC e visam quantificar os danos oxidativos às macromoléculas (DNA, lipídeos e proteínas) em amostras biológicas in vivo ou ex vivo (Moret-Tatay, 2016). Esses marcadores biológicos são empregados em função da meia-vida extremamente curta das EROs, sendo de grande complexidade mensurá-las diretamente (Halliwell, 2007).

Nos estados catabólicos da DC, onde há aumento do estado inflamatório, há incremento de alguns desses biomarcadores, evidenciando falha nos sistemas de defesa antioxidante (Bourgonje, 2019). Esse quadro se agrava em função da má absorção ou absorção prejudicada de antioxidantes dietéticos. Com a falha nos sistemas antioxidantes, perde-se a capacidade de impedimento da geração das EROs e dos seus efeitos oxidativos em cascata.

Além disso, as ações de proteção contra o surgimento de danos oxidativos às proteínas (carbonilação ou nitração), aos lipídeos (lipoperoxidação e formação de peróxidos lipídicos) e às bases do DNA (oxidação de nucleotídeos) ficam limitadas (Halliwell, 2007). O controle desses eventos no paciente DC é muito necessário, uma vez que podem acarretar em perdas da integridade celular. Por exemplo, na oxidação lipídica, ocorrem alterações na fluidez das membranas celulares, com a entrada de substâncias tóxicas no meio intracelular, podendo causar sua lise (Barbosa et al, 2010).

Nesse sentido, as substâncias reativas ao ácido tiobarbitúrico (TBARS) são um dos principais biomarcadores. Além do TBARS, outro importante marcador de lipoperoxidação (LPO) são os peróxidos lipídicos (PL). Os PLs têm ampla 
capacidade de ação, alcançando diversos alvos biológicos (Barbosa et al, 2010). Estão envolvidos nas reações de ataque aos PUFA dos fosfolipídeos de membrana, causando degradação e alteração no controle de fluxo celular (Freinbichler et al, 2011). Além do ataque aos PUFA, também pode ocorrer produção concomitante de citocinas inflamatórias, como já demonstrado em células epiteliais intestinais de pacientes DC (Mayr et al, 2020).

$\mathrm{Na}$ avaliação do grau de oxidação proteica, o biomarcador mais utilizado é proteína carbonilada (PC) em função de sua estabilidade e fácil determinação a partir do reagente DNPH (Shearn et al, 2017). As proteínas são alvos fáceis para lesões oxidativas, já que estão amplamente distribuídas em nível plasmático, sérico e tecidual. As principais consequências da oxidação proteica incluem alterações na função de receptores, enzimas, moléculas transdutoras de sinal ou transportadoras, dentre outras. Os produtos dessa oxidação são reconhecidos como antígenos pelo sistema imunológico, ativando a produção de anticorpos e gerando uma reação autoimune (Freinbichler et al, 2011). Esses eventos são sabidamente implicados na DC.

No que se refere ao sistema de defesa antioxidante endógeno, os principais mediadores envolvidos na DC são do grupo das Glutationas (Glutationa Redutase, GR; Glutationa-S-transferase, GST e Glutationa Peroxidase- GPx). A enzima GPx, em dependência da oxidação simultânea da GR, age protegendo as células contra altas concentrações de $\mathrm{H}_{2} \mathrm{O}_{2}$ reduzindo o mesmo à água (Minich \& Brown, 2019).

A glutationa é produzida endogenamente a partir da disponibilidade de três aminoácidos (cisteína, glicina e glutamina) e é fundamental no combate das EROs, sendo importante para eliminar as toxinas do organismo (Smits et al, 2019).

Dentre os antioxidantes exógenos, o $\beta$-caroteno é um dos de mais fácil obtenção, porém em função de ser uma molécula lipofílica, apresenta absorção dificultada em pacientes DC ativos. Pertencente ao grupo dos carotenoides, é encontrado nas frutas de cor amarelo alaranjada e em vegetais folhosos verde escuro (Langi et al, 2018). Após a absorção intestinal, é convertido a retinol (60 a 70\%) e metabolizado. Seu transporte é feito no plasma por meio das lipoproteínas e seus locais de armazenamento são os tecidos hepático e adiposo.

O selênio, outro exemplo de molécula antioxidante dietética, também tem sido estudado na DC, especialmente por estar envolvido com a remediação da inflamação e do estresse oxidativo. Trata-se de um mineral (oligoelemento) relacionado com a minimização do risco de doenças crônicas causadas por processos oxidativo-inflamatórios (Geraldo \& Alfenas, 2008; Barros et al, 2020).

Especificamente o selênio age impedindo a ativação do fator de transcrição Nuclear Kappa-beta (NF-к $\beta$ ), que é um regulador sensível a oxidantes que modula a síntese de mediadores inflamatórios e de moléculas de adesão (Volp et al, 2010). Além disso, sua ação inibitória sobre a liberação de citocinas pró-inflamatórias como a Interleucina-6 (IL-6) e o Fator de Necrose Tumoral- $\alpha$ (TNF- $\alpha$ ) já foi demonstrada (Volp et al, 2010).

\section{Discussão}

A Tabela 2 resume os principais marcadores de estresse oxidativo e antioxidantes em pacientes com DC. 
Tabela 2 - Alterações nos marcadores de estresse oxidativo e defesas antioxidantes em pacientes DC.

\begin{tabular}{|c|c|c|c|c|}
\hline Biomarcador & Amostra & População estudada & Resultados & Referências \\
\hline TBARS, PL, $\beta$-caroteno e GPx & Soro & 16 pacientes DC ativos, 27 & $\begin{array}{c}\uparrow \text { TBARS, PL e GPx em pacientes DC } \\
\text { ativos; } \downarrow \beta \text {-caroteno em ativos e ER }\end{array}$ & Maor et al, 2008 \\
\hline & & ER e 15 controles & & \\
\hline SOD1, GPx, and CAT & Eritrócitos & 53 pacientes DC ativos, 28 & $\downarrow$ CAT e GPx & $\begin{array}{l}\text { Krzystek-Korpacka } \\
\text { et al, } 2010\end{array}$ \\
\hline & & ER e 105 controles & & \\
\hline Selênio & Soro & 351 pacientes DC e 853 controles & $\downarrow$ selênio & $\begin{array}{c}\text { Gentschew et al, } \\
2012\end{array}$ \\
\hline TAC, TOS & Soro & 40 pacientes DC & $\downarrow \mathrm{TAC}$ e $\uparrow \mathrm{TOS}$ & Yuksel et al, 2017 \\
\hline FRAP, GSH & Plasma & $\begin{array}{c}30 \text { pacientes DC ativos, } 25 \text { ER e } 25 \\
\text { controles }\end{array}$ & $\downarrow$ FRAP e GSH em DC ativos & $\begin{array}{l}\text { Szczeklik et al, } \\
2018\end{array}$ \\
\hline TBARS, PC & Plasma & 54 pacientes DC e 17 controles & $\uparrow$ TBARS e PC em pacientes DC & Luceri et al, 2019 \\
\hline PS & Plasma & 50 pacientes DC & $\downarrow$ PS & $\begin{array}{l}\text { Bourgonje et al } \\
2019\end{array}$ \\
\hline PS, TAC & Soro & 47 pacientes DC & $\downarrow$ PS e TAC & Neubauer et al, 2019 \\
\hline GPx, Selênio e TBARS & $\begin{array}{l}\text { Plasma e } \\
\text { eritrócitos }\end{array}$ & $\begin{array}{c}20 \text { pacientes DC ativos, } 27 \text { ER e } 25 \\
\text { controles }\end{array}$ & $\downarrow$ selênio; $\uparrow$ TBARS e GPx & Barros et al, 2020 \\
\hline
\end{tabular}

Legenda: CAT (catalase); DC (doença de Crohn); ER (em remissão; paciente estável ou inativo); FRAP (capacidade redutora de ferro plasmático); GPx (glutationa peroxidase); TBARS (produtos de reação ao ácido tiobarbitúrico); PC (proteínas carboniladas); PL (peróxidos lipídicos); PS (proteína sulfidril); SOD1 (superóxido dismutase 1); TAC (capacidade antioxidante total); TOS (status oxidante total). Fonte: Autores. 
Como é possível observar na Tabela 2, a maioria dos estudos aborda marcadores de danos oxidativos aos lipídeos, indicando que os níveis séricos aumentados de TBARS e/ou de peróxidos lipídicos (PL), estão fortemente associados à DC.

De acordo com o estudo de Maor et al (2008), foi encontrado um aumento de TBARS no soro de pacientes ativos superior em cerca de 1,3 vezes ao exibido por indivíduos remissivos e 2 vezes comparado aos controles. Quando a comparação foi feita nesses últimos, esse aumento foi de 1,5 vezes para os remissivos. Resultados semelhantes foram descritos pelo mesmo autor para os níveis de peróxidos lipídicos (PL), em que os pacientes ativos apresentaram aumento de 1 vez em relação aos remissivos e 1,3 vezes perante aos controles.

Ainda em relação aos marcadores lipídicos, no estudo de Luceri et al (2019) em que foram incluídos 54 pacientes com DC, foi demonstrado um aumento nos níveis de TBARS plasmático dos pacientes de cerca de 1,3 vezes em relação aos controles. Ainda, relatou-se um aumento expressivo de $65 \%$ nos níveis de proteínas carboniladas (PC), um importante marcador que avalia oxidação proteica. Esses resultados foram corroborados pelo estudo de Barros e colaboradores (2020), os quais reportaram um aumento de cerca de 1,3 vezes para níveis de TBARS em pacientes ativos comparados aos remissivos e de 1,5 vezes quando comparados os ativos aos controles.

A glutationa (GSH), o tiol de baixo peso molecular mais abundante no corpo humano, é um marcador sensível de defesa antioxidante. Medidas periféricas no soro, plasma e eritrócitos já demonstraram alterações dos níveis circulantes de GSH e/ou da atividade da GPx em pacientes com DC.

De acordo com o estudo de Krzystek-Korpacka et al (2010), os pacientes ativos apresentaram redução de $38 \%$ nos níveis de GPx eritrocitário em relação ao grupo controle e 31,5 \% em comparação ao grupo ER. Esses últimos exibiram redução de cerca de 9,5\% quando comparados aos controles. Esses achados foram corroborados em estudo recente envolvendo 55 pacientes com DC, dos quais 30 encontravam-se em fase ativa da doença e 25 ER (Szczeklik et al, 2018). No estudo em questão, foi encontrado uma redução significativa (cerca de 2 vezes) nos níveis de GSH de pacientes ativos em relação ao controle. Com relação aos pacientes ER, no entanto, não foi encontrada diferença estatística. Esses achados divergem dos encontrados por Maor et al (2008) e Barros et al (2020). Nesse último, um aumento de 43 e $45 \%$ na atividade de GPx eritrocitária foi reportado em pacientes ativos e remissivos, respectivamente, quando comparados aos controles. Na comparação de pacientes ativos e ER os níveis foram semelhantes $(53,45 \pm 15,81$ e 54,8 $\pm 11,43$, respectivamente). Já no estudo de Maor et al (2008), a atividade enzimática de GPx foi aumentada em 31 \% quando comparados os pacientes ativos aos controles, e esse aumento foi explicado como uma tentativa de combater o estresse oxidativo desencadeado pelo processo inflamatório vigente da doença.

Assim como a GPx, a CAT também atua na minimização do estresse oxidativo através da detoxificação do peróxido de hidrogênio $\left(\mathrm{H}_{2} \mathrm{O}_{2}\right)$. Embora não sendo uma espécie radicalar, o mesmo está envolvido na produção do radical hidroxila ('OH), uma espécie altamente reativa e instável, não havendo antioxidantes enzimáticos capazes de eliminá-lo. Dessa forma, minimizar a geração de $\mathrm{H}_{2} \mathrm{O}_{2}$ é o primeiro passo para evitar a sua produção.

No estudo de Krzystek-Korpacka et al (2010), observou-se a redução da atividade da CAT em ambos pacientes ativos e ER. Nos 53 pacientes com DC ativa relatou-se uma diminuição significativa de $20 \%$ em relação aos controles, sendo que essa redução foi de apenas 9\% no grupo remissivo, sem diferença estatística.

O único estudo da presente revisão que avaliou o marcador SOD na DC não encontrou diferença estatística significativa entre os grupos ativo, remissivo e controle (Krzystek-Korpacka et al, 2010). No entanto, foi demonstrado que a atividade de SOD1 se correlacionou negativamente com o índice de atividade clínica da doença $(r=-0,290 ; p=0,025)$.

Além das defesas enzimáticas, marcadores não enzimáticos também parecem estar significativamente alterados na DC. Na pesquisa de Barros et al (2020), foi analisado o selênio no plasma de 20 pacientes com DC ativa e 27 ER comparados 
a um grupo controle $(\mathrm{n}=25)$. Verificou-se, nos pacientes ativos, uma redução plasmática de cerca de $30 \%$ em relação ao controle, enquanto que pacientes ER exibiram uma taxa $22 \%$ menor. Esses achados foram semelhantes aos descritos previamente por Gentschew et al (2012), os quais reportaram em 351 pacientes uma redução de 8,4 \% se comparado ao grupo controle. Os autores destacaram em seu estudo que essa diminuição está associada ao desenvolvimento de outros problemas de saúde, tais como distúrbios neurológicos.

Além dos níveis de selênio, o micronutriente $\beta$-caroteno também exibiu níveis séricos diminuídos nos pacientes DC (redução de $66 \%$ ) em relação ao controle, sendo que quando comparados apenas pacientes remissivos e ativos, foi relatado uma redução de $48 \%$ nesses últimos (Maor et al, 2008).

Tomadas em conjunto, as defesas antioxidantes colaboram para a manutenção do equilíbrio redox do organismo. Marcadores globais de defesa, tais como a capacidade antioxidante total (TAC) demonstraram-se significativamente reduzidos na DC, como previamente demonstrado no estudo de Yuksel et al (2017). No estudo em questão, foi reportada uma redução de cerca de $32 \%$ na TAC sérica de pacientes ativos, quando comparados aos indivíduos do grupo controle. Esse achado foi corroborado em estudo recente (Neubauer et al, 2019), em que foram comparados 47 pacientes DC e 57 indivíduos controle, tendo sido observada uma redução de quase $20 \%$ na TAC do primeiro grupo.

Além da TAC, outro importante marcador global de estresse oxidativo é o status oxidante total (TOS). Em seu estudo, Yuksel et al (2017) reportaram um aumento de 45 \% nos níveis desse biomarcador em pacientes com DC em comparação aos controles saudáveis.

Outro biomarcador importante que tem relação com TOS é o FRAP (Ferric Reducing Antioxidant Power) e baseia-se na redução do ferro plasmático como uma medida indireta de estresse oxidativo. De acordo com o estudo de Szczeklik et al (2018), esse marcador foi significativamente alterado na DC, especificamente em $50 \%$ na comparação de pacientes ativos com controles, sendo que essa redução foi de apenas $10 \%$ quando comparado o grupo ER com o controle.

Reunindo todas essas evidências, é possível observar que o monitoramento desses biomarcadores na população portadora de DC ativa e/ou remissiva pode representar uma abordagem bastante útil para avaliar o balanço oxidativo sistêmico dos pacientes e estabelecer estratégias de tratamento em caso de desequilíbrio.

Os dados aqui mostrados destacam que, mesmo com a doença sob controle, os pacientes remissivos continuam a apresentar níveis de estresse oxidativo altos e defesas antioxidantes alteradas, demonstrando que a medicação convencional não exerce influência sobre esses parâmetros, sendo necessárias novas estratégias adjuvantes, como o aporte de vitaminas e minerais com ação antioxidante, seja por meio da dieta ou via suplementação.

\section{Conclusões e Perspectivas Futuras}

Existem evidências satisfatórias que apontam para a modulação positiva intestinal exibida pelos antioxidantes dietéticos, principalmente devido ao seu efeito anti-inflamatório. A melhora do status redox no microambiente intestinal reduz o estresse oxidativo associado à inflamação, melhorando os parâmetros bioquímicos e suas consequências, assim contribuindo na redução dos custos de saúde incorridos nos pacientes portadores de DC. No entanto, apesar de todas as evidências apresentadas nessa revisão, novas pesquisas são necessárias para avaliar os efeitos dos antioxidantes dietéticos, em pacientes ativos e remissivos, bem como o monitoramento de biomarcadores de estresse oxidativo em estudos longitudinais.

\section{Referências}

ABCD - Associação Brasileira de Colite Ulcerativa e Doença de Crohn. (2020). http://www.abcd.org.br/. 
Alatab, S., Sepanlou, S. G., Ikuta, K.; Vahedi, H., Bisiganano, C., Safiri, S., Sadeghi, A., Nixon, M. R., Abdoli, A. \& Abolhassani, H. (2020). The global, regional, and national burden of inflammatory bowel disease in 195 countries and territories, 1990-2017: a systematic analysis for the global burden of disease study 2017. The Lancet Gastroenterology \& Hepatology, 5(1), 17-30.

Aleksandrova, K., Romero-Mosquera, B., \& Hernandez, V. (2017). Diet, Gut Microbiome and Epigenetics: emerging links with inflammatory bowel diseases and prospects for management and prevention. Nutrients, 9(9), 17-30.

Alemany-Cosme, E., Sáez-Gonzáles, E., Moret, I., Mateos, B., Iborra, M., Nos, P., Sandoval, J., Beltrán, B. (2021). Oxidative Stress in the Pathogenesis of Crohn's Disease and the Interconnection with Immunological Response, Microbiota, External Environmental Factors, and Epigenetics. Antioxidants, 10(1), 121.

Alzoghaibi, M. A. (2013) Concepts of oxidative stress and antioxidant defense in Crohn's disease. World Journal of Gastroenterology, 19 (39), 6540-6547.

Ananthakrishnan, A. N. (2015). Epidemiology and risk factors for IBD. Nature Reviews Gastroenterology \& Hepatology, 12 (4), $205-217$.

Barbosa, K. B. F., Costa, N. M. B., Alfenas, R. C. G., Paula, S. O., Minim, V. P. R. \& Bressan, J. (2010). Estresse oxidativo: conceito, implicações e fatores modulatórios. Revista de Nutrição, 23(4), 629-643.

Barros, S. E. L., Dias, T. M. S., Moura, M. S. B., Soares, N. R. M., Pierote, N. R. A., Araújo, C. O. D., Maia, C. S. C., Henriques, G. S., Barros, V. C., Neto, J. M. M., Parente, J. M. L., Marreiro, D. N. \& Nogueira, N. N. (2020). Relationship between selenium status and biomarkers of oxidative stress in Crohn's disease. Nutrition, 74, 1-5.

Bourgonje, A. R., Martels, J. Z. H., Bulthuis, M. L. C., Londen, M., Faber, K. N., Dijkstra, G. \& Goor, H. (2019). Crohn's Disease in Clinical Remission Is Marked by Systemic Oxidative Stress. Frontiers in Physiology, 10, 1-10.

Branco, C. S., Lima, E. D., Rodrigues, T. S., Scheffel, T. B., Scola, G., Laurino, C. C. F. C., Moura, S. \& Salvador, M. (2015). Mitochondria and redox homoeostasis as chemotherapeutic targets of Araucaria angustifolia (Bert.) O. Kuntze in human larynx HEp-2 cancer cells. Chemico-Biological Interactions, $231,108-118$

Brown, A. C., Rampertab, S. D. \& Mullin, G. E. (2011). Existing dietary guidelines for Crohn's disease and ulcerative colitis. Expert Review of Gastroenterology \& Hepatology, 5(3), 411- 425.

Cavalcante, R. M. S. (2020). Inflammatory Bowel Diseases and diet: an integrative review. Revista da Associação Médica Brasileira, 66(10), 1449-1454.

Chan, W. P. W., Mourad, F. \& Leong, R. W. (2018). Crohn's disease associated strictures. Journal of Gastroenterology and Hepatology, 33(5), 998-1008.

Errante, P. R. \& Romano Júnior, S. C. (2016). Doença de Crohn, diagnóstico e tratamento. Atas de Ciências da Saúde, 4(4), 31-50.

Ferré, M. P. B., Boscá-Watts, M. M. \& Pérez, M. M. (2018). Enfermedad de Crohn. Medicina Clínica, 151(1), 26-33.

Flynn, S. \& Eisenstein, S. (2019). Inflammatory Bowel Disease Presentation and Diagnosis. Surgical Clinics of North America, 99(6), 1051-1062.

Freinbichler, W., Colivicchi, M. A., Stefanini, C. S., Bianchi, L., Ballini, C., Misini, B., Weinberger, P., Linert, W., Varešlija, D., Tipton, K. F. \& Corte, L. D. (2011). Highly reactive oxygen species: detection, formation, and possible functions. Cellular and Molecular Life Sciences, 68(12), $2067-2079$.

Gentschew, L., Bishop, K. S., Han, D. Y., Morgan, A. R., Fraser, A. G., Lam, W. J., Karunasinghe, N., Campbell, B. \&Ferguson, L. R. (2012). Selenium, Selenoprotein Genes and Crohn's Disease in a Case-Control Population from Auckland. Nutrients, 4(9), $1247-1259$.

Geraldo, J. M. \& Alfenas, R. C. G. (2008). Papel da dieta na prevenção e no controle da inflamação crônica: evidências atuais. Arquivos Brasileiros de Endocrinologia \& Metabologia, 52(6), 951-967.

Goens, D. \& Micic, D. (2020). Role of Diet in the Development and Management of Crohn's Disease. Current Gastroenterology Reports, 22(4), 1-9.

Goodman, W. A., Eekkila, I. P. \& Pizarro, T. T. (2020). Sex matters: impact on pathogenesis, presentation and treatment of inflammatory bowel disease. Nature Reviews Gastroenterology \& Hepatology, 17(12), 740-754.

Guan, G. \& Lan, S. (2018). Implications of Antioxidant Systems in Inflammatory Bowel Disease. Biomed Research International, $2018,1-7$.

Halliwell, B. (2007). Biochemistry of oxidative stress. Biochemical Society Transactions, 35(5), 1147-1150.

Hendy, P. \& Hart, A. (2013). A review of Crohn's disease. European Medical Journal - Gastroenterology, 1, 116-123.

Krzystek-Korpacka, M., Neubauer, K., Berdowska, I., Zielinski, B., Paradowski, L. \& Gamian, A. (2010). Impaired erythrocyte antioxidant defense in active inflammatory bowel disease: impact of anemia and treatment. Inflammatory Bowel Diseases, 16(9), 1467-1475.

Kumar, M., Hemalatha, R., Nagpal, R., Singh, B., Parasannanavar, D., Verma, V., Kumar, A., Marotta, F., Catanzaro, R., Cuffari, B., Jain, S., Bissi, L. \& Yadav, H. (2016). Probiotic approaches for targeting inflammatory bowel disease: an update on advances and opportunities in managing the disease. International Journal of Probiotics \& Prebiotics, 11(3-4), 99-116.

Langi, P., Kiokias, S., Varzakas, T. \& Proestos, C. (2018). Carotenoids: from plants to food and feed industries. Methods in Molecular Biology, 1852, 57-71.

Luceri, C., Bigagli, E., Agostiniani, S., Giudici, F., Zambonin, D., Scaringi, S., Ficari, F., Lodovici, M. \& Malentacchi, C. (2019). Analysis of Oxidative Stress-Related Markers in Crohn's Disease Patients at Surgery and Correlations with Clinical Findings. Antioxidants, 8(9), 1-12. 
Lichtenstein, Gary R., Loftus, E. V., Isaacs, K. L., Regueiro, M. D., Gerson, L. B. \& Sands, B. E. (2018). ACG Clinical Guideline: management of Crohn's disease in adults. American Journal of Gastroenterology, 113(4), 481-517.

Maor, I., Raini, T., Lanir, A. \& Lavy, A. (2008). Oxidative stress, inflammation and neutrophil superoxide release in patients with Crohn's disease: distinction between active and non-active disease. Digestive Diseases and Sciences, 53(8), 2208-2214.

Mariosa, F. G., Chebli, J. M. F. \& Chebli, L. A. (2011). Supervisão e manejo pós-operatório na Doença de Crohn. Hu Revista. 37(2), $233-238$.

Mayr, L., Grabherr, F. Schwärzler, J., Reitmeier, I., Sommer, F., Gehmacher, T., Niederreiter, L., He, G. W., Ruder, B., Kunz, K. T. R., Tymoszuk, P., Hilbe, R., Haschka, D., Feistritzer, C., Gerner, R. R., Enrich, B., Przysiecki, N., Seifert, M., Keller, M. A., Oberhuber, G., Sprung, S., Ran, Q., Koch, R., Effenberger, M., Tancevski, I., Zoller, H., Moschen, A. R., Weiss, G., Becker, C., Rosenstiel, P., Kaser, A., Tilg, H. \& Adolph, T. E. (2020). Dietary lipids fuel GPX4restricted enteritis resembling Crohn's disease. Nature Communications, 11(1775), 1-15.

Minich, D. M. \& Brown, B. I. (2019). A Review of Dietary (Phyto)Nutrients for Glutathione Support. Nutrients, 11(9), 1-20.

Moret-Tatay, I., Iborra, M., Cerrillo, E., Tortosa, L., Nos, P. \& Beltrán, B. (2016). Possible biomarkers in blood for Crohn's disease: oxidative stress and microRNAs - current evidences and further aspects to unravel. Oxidative Medicine and Cellular Longevity, 2016, 1-9.

Neha, K., Haider, M. R., Pathak, A. \& Yar, M. S. (2019). Medicinal prospects of antioxidants: a review. European Journal of Medicinal Chemistry, 178, 687704.

Neurath, F. M. (2019). Targeting immune cell circuits and trafficking in inflammatory bowel disease. Nature Immunology, 20(8), 970-979.

Neubauer, K., Kempinski, R., Matusiewicz, M., Bednarz-Misa, I. \& Krzystek-Korpacka, M. (2019). Nonenzymatic Serum Antioxidant Capacity in IBD and Its Association with the Severity of Bowel Inflammation and Corticosteroids Treatment. Medicina (Kaunas), 55(4), 1-14.

Ng, S. C., Shi, H. Y., Hamidi, N., Underwood, F. E., Tang, W., Benchimol, E. I., Panaccione, R., Gohsh, S., Wu, J. C. Y., Chan, F. K.L., Sung, J. J. Y. \& Kaplan, G. G. (2017). Worldwide incidence and prevalence of inflammatory bowel disease in the 21st century: a systematic review of population-based studies. The Lancet, 390(10114), 2769-2778.

Owczarek, D., Rodacki, T., Domagala-Rodacka, R., Cibor, D. \& Mach, T. (2016). Diet and nutritional factors in inflammatory bowel diseases. World Journal of Gastroenterology, 22(3), 895-905.

Pariente, B., Cosnes, J., Danese, S., Sandborn, W. J., Lewin, M., Fletcher, J. G., Chowers, Y., D'Haens, G., Feagan, B. G., Hibi, T., Hommes, D. W., Irvine, E. J., Kamm, M. A., Loftus, E. V., Michetti, E. L. P., Munkholm, P., Oresland, T., Panés, J., Peyrin-Biroulet, L., Reinisch, W., Sands, B. E., Schoelmerich, J., Schreiber, S., Tilg, H., Travis, S., Assche, G., Vecchi, M., Mary, J., Colombel, J. \& Lémann, M. (2011) Development of the Crohn's disease digestive damage score, the Lémann score. Inflammatory Bowel Diseases, 17(6), 1415-1422.

Petagna, L., Antonelli, A., Ganini, C., Bellato, V., Campanelli, M., Divizia, A., Efrati, C., Franceschilli, M., Guida, A. M., Ingallinella, S., Montagnese, F. Sensi, B., Siragusa, L., \& Sica, G. S. (2020). Pathophysiology of Crohn's disease inflammation and recurrence. Biology Direct, 15(1), 1-10.

Pizzino, G., Irrera, N., Cucinotta, M., Pallio, G., Mannino, F., Arcoraci, V., Squadrito, F., Altavilla, D. \& Bitto, A. (2017) Oxidative Stress: harms and benefits for human health. Oxidative Medicine and Cellular Longevity, 2017, 1-13.

Ramos, G. P. \& Papadakis, K. A. (2019). Mechanisms of Disease: inflammatory bowel diseases. Mayo Clinic Proceedings, 94(1), 155-165.

Reddavide, R., Rotolo, O., Caruso, M. G., Stasi, E., Notarnicola, M., Miraglia, C., Nouvenne, A., Meschi, T., Angelis, G. L., Mario, F. \& Leandro, G. (2018). The role of diet in the prevention and treatment of Inflammatory Bowel Diseases. Acta Bio Medica Atenei Parmensis, 89(9), 60-75.

Roda, G., Ng, S. C., Kotze, P. G., Argollo, M., Panaccione, R., Spinelli, A., Kaser, A., Peyrin-Biroulet, L. \& Danese, S. (2020). Crohn's disease. Nature Reviews Disease Primers, 6(1), 1-19.

Sairenji, T., Collins, K. L. \& Evans, D. V. (2017). An Update on Inflammatory Bowel Disease. Primary Care: Clinics in Office Practice, 44(4), 673-692.

Santarpia, L., Alfonsi, L., Castiglione, F., Pagano, M. C., Cioffi, I., Rispo A., Sodo, M., Contaldo, F. \& Pasanisi, F. (2019). Nutritional Rehabilitation in Patients with Malnutrition Due to Crohn's Disease. Nutrients, 11 (12), 1-9.

Seyedian, S. S., Nokhostin, F. \& Malamir, M. D. (2019). A review of the diagnosis, prevention, and treatment methods of inflammatory bowel disease. Journal of Medicine and Life, 12(2), 113-122.

Shearn, C. T., Saba, L. M., Roede, J. R., Orlicky, D. J., Shearn, A. H. \& Petersen, D. R. (2017). Differential carbonylation of proteins in end-stage human fatty and nonfatty NASH. Free Radical Biology and Medicine, 113, 280-290.

Sies, H. (2018). On the history of oxidative stress: concept and some aspects of current development. Current Opinion in Toxicology, 7, 122-126.

Smits, R. M., Mackenzie-Proctor, R., Yazdani, A., Stankiewicz, M.T., Jordan, V. \& Showell, M.G. (2019). Antioxidants for male subfertility. The Cochrane Database of Systematic Reviews, 3(3), 1-4.

Spiller, R. C. \&Thompson, W. G. (2012). Transtornos intestinais. Arquivos de Gastroenterologia, 49, supl. 1, 39-50.

Szczeklik, K., Krzyściak, W., Cibor, D., Kozioł, K., Pocztar, J., Pytko-Polończyk, J., Mach, T. \& Owczarek, D. (2018). Evaluation of plasma concentrations of selected antioxidant parameters in patients with active Crohn's disease. Folia Medica Cracoviensia, 58(2), 119-130.

Torres, J., Mehandru, S., Colombel, J. F., Peyrin-Biroulet, L. (2017). Crohn's disease. The Lancet, 389(10080), 1741-1755. 
Research, Society and Development, v. 10, n. 4, e52910414445, 2021

(CC BY 4.0) | ISSN 2525-3409 | DOI: http://dx.doi.org/10.33448/rsd-v10i4.14445

Tumani, M. F., Pavez, C. \& Parada, A. (2020). Microbiota, hábitos alimentarios y dieta en enfermedad inflamatoria intestinal. Revista Chilena de Nutrición, $47(5), 822-829$

Veauthier, B. \& Hornecker, J. R. (2018). Crohn's Disease: Diagnosis and Management. American Family Physician, 98(11), 661-669E.

Volp, A. C. P., Bressan, J., Hermsdorff, H. H. M., Zulet, M. A. \& Martínez, J. A. (2010). Efeitos antioxidantes do selênio e seu elo com a inflamação e síndrome metabólica. Revista de Nutrição, 23(4), 581-590.

Warraich, U. A., Hussain, F. \& Kayani, H. U. R. (2020). Aging-Oxidative stress, antioxidants and computational modeling. Heliyon, 6(5), 1-11.

Weisshof, R. \& Chermesh, I. (2015). Micronutrient deficiencies in inflammatory bowel disease. Current Opinion in Clinical Nutrition and Metabolic Care, 18(6), 576-581.

Wright, E., K, Kamm, M. A., Teo, S. M., Inouye, M., Wagner., J. \& Kirkwood, C. D. (2015). Recent Advances in Characterizing the Gastrointestinal Microbiome in Crohn's Disease. Inflammatory Bowel Diseases, 6(21), 1219-1228.

Yuksel, M., Ates, I., Kaplan, M., Arikan, M, F., Ozin, Y. O., Kilic, Z. M. Y., Topcuoglu, C. \& Kayacetin, E. (2017). Is Oxidative Stress Associated with Activation and Pathogenesis of Inflammatory Bowel Disease? Journal Of Medical Biochemistry, 36(4), 341-348. 